\title{
Um arcabouço conceitual para o estudo da trajetória de desenvolvimento em territórios: contribuições da mudança institucional e dos Commons
}

\author{
A conceptual framework for the study of territorial development trajectory: contributions of \\ institutional change and Commons
}

Rodrigo Rodrigues de Freitas ${ }^{1}$

\begin{abstract}
Resumo
O estudo da trajetória de desenvolvimento vem sendo amplamente utilizado nas pesquisas sobre desenvolvimento territorial sustentável. No entanto, as pesquisas empíricas sobre a trajetória de desenvolvimento carecem de referenciais analíticos que permitam avançar no estudo de padrões e processos comuns aos casos. Nesse sentido, proponho um arcabouço conceitual que oferece a possibilidade empírica de realizar estudos sobre a trajetória de desenvolvimento em estreita sintonia com a análise das mudanças institucionais processadas em recursos naturais de uso comum. $\mathrm{O}$ arcabouço conceitual é focado nas interações entre variáveis que atuam na mudança institucional: vetores de mudança, inovações técnicas, consequências para os recursos naturais e nas complexas relações entre as regras formais e informais de acesso e uso de recursos naturais. A mudança institucional é direcionada pelas interações entre as organizações, analisadas através do jogo de atores, que pode ser operacionalizada pelo uso de modelos auxiliares. $\mathrm{O}$ arcabouço conceitual sugere a busca por complementaridades entre as categorias de sistema socioecológico e território e foi fruto de um estudo sobre a pesca artesanal na Baía da Ilha Grande (Rio de Janeiro, Brasil). A abordagem proposta busca inserir a gestão de recursos naturais de uso comum em um cenário de desenvolvimento territorial sustentável e contribuir para os avanços nas pesquisas sobre o fenômeno territorial do desenvolvimento.
\end{abstract}

Palavras-chave: Mudança institucional. Recursos de uso comum. Arcabouço conceitual. Desenvolvimento territorial.

\begin{abstract}
The study of development trajectory has been widely used in researches about sustainable territorial development. However, empirical researches about the development trajectory lack analytical references to advance in the study of common patterns and processes to cases. In this sense, I purpose a conceptual framework that offer an empirical possibility to conduct studies about development trajectory in close tuning with institutional changes analysis processed in common use natural resources. The framework is focused in the feedbacks between variables that act in the institutional change: drives of change, technical innovations, consequences for natural resources and in the complex relationship between formal and informal rules on access to and use of natural resources. The institutional change is driven by interactions between organizations, analyzed
\end{abstract}

1Doutor em Ambiente e Sociedade (ÚNICAMP). Professor da Universidade do Sul de Santa Catarina (UNISUL). Email: rodrigo.manejo@gmail.com. 
through stakeholder's interaction, which can be operationalized by the use of auxiliary models. The conceptual framework suggests the search by complementarities between the categories of socioecological system and territory and was the result of a study about small-scale fisheries in Ilha Grande Bay (Rio de Janeiro, Brazil). The purpose approach search to insert the management of natural common resources in a sustainable territorial development scenario, and contribute for advances in researches about the territorial development phenomena.

Keywords: Institutional change. Common use resources. Conceptual framework. Territorial development.

\section{Introdução}

A trajetória de desenvolvimento foi concebida como uma proposta de abordagem da dinâmica rural (SABOURIN; CARON; SILVA, 1996; SABOURIN, 2001), sendo imprescindível para o estudo do desenvolvimento na sua configuração territorial (VIEIRA; CAZELLA, 2006; VIEIRA, 2006). Essa abordagem se apoia em métodos de análise de transformações técnicas, econômicas e sociais articuladas em diferentes escalas temporais e espaciais (SABOURIN; CARON; SILVA, 1996). O estudo da trajetória de desenvolvimento de territórios se baseia na recuperação da memória coletiva e na identificação da evolução dos processos de inovação (SABOURIN, 2001). Segundo Vieira (2006), a trajetória de desenvolvimento deve desvelar as estruturas de dominação, as lógicas específicas de ação coletiva e as conexões institucionais transescalares que não estão presentes no diagnóstico explicativo tradicional de operações de desenvolvimento.

A problemática da ação coletiva está associada com o desafio de evoluir de uma situação dominada por indivíduos agindo exclusivamente em função de interesses pessoais para outra, na qual prevalece a busca de maiores benefícios e menores custos para todos os envolvidos (VIEIRA, 2006). As conexões transescalares ressaltam o fato de que, quando há ligações entre as escalas, os problemas de gestão devem ser tratados em diversas escalas simultaneamente, e não de forma isolada (BERKES, 2002).

Segundo Sabourin, Silva e Caron (1996), os estudos sobre trajetória de desenvolvimento têm as seguintes utilidades: (1) produção de indicadores de mudança; (2) identificação de temas privilegiados de intervenção para o desenvolvimento em termos de organização dos atores em torno da apropriação da terra, da experimentação técnica e do acesso ao mercado; e (3) contribuição para 
a formulação de cenários de desenvolvimento levando em consideração a articulação entre as escalas.

Os estudos sobre trajetória de desenvolvimento de territórios realizados no Brasil não possuem categorias analíticas predefinidas. Cerdan et al. (2011) utilizaram o modelo DPSR (Driving forces, Pressões, Estado e Respostas), “geralmente mobilizado para organizar indicadores ambientais" (Ibid., p. 21). A trajetória de desenvolvimento elaborada por esses autores teve o objetivo de identificar as dinâmicas territoriais em curso. A trajetória de desenvolvimento elaborada por Flores (2007) trata de mudanças institucionais como novas políticas e legislações para um ramo da indústria (vitinicultura) ancoradas espacialmente (Vale dos Vinhedos), havendo pouca ou nenhuma intencionalidade de extrapolar os resultados em termos de padrões e processos para outros contextos. Andion (2007) enfoca a trajetória de desenvolvimento nas organizações e no ambiente em que ocorre o desenvolvimento, partindo de uma perspectiva sincrônica e diacrônica.

Este artigo tem o objetivo de apresentar um arcabouço analítico para o estudo da trajetória de desenvolvimento de territórios usando a teoria da mudança institucional. As instituições são entendidas como as regras do jogo em uma sociedade ou as restrições que moldam a interação humana (NORTH, 1990). Elas são formadas por restrições formais, restrições informais e as características dos mecanismos de fazer cumprir as regras. Nesse sentido, instituições sociais são sistemas de regras (OSTROM; BASURTO, 2011), pois as regras estão envolvidas em contextos sociais, econômicos ou políticos e formam um sistema coerente, em vez de uma simples aglomeração (CARRUTHERS, 2012).

As pesquisas sobre mudança institucional vêm recebendo destaque em análises sobre a governança dos recursos de uso comum (e.g. OSTROM; BASURTO, 2011) desde que se constatou que a ênfase nas estruturas institucionais limitava a compreensão da dinâmica dos sistemas (AGRAWAL, 2002). Estudos sobre dinâmicas territoriais que utilizam teorias sobre mudança institucional e ação coletiva (e.g. BERDEGUÉ et al., 2012) conferem visibilidade às variáveis ambientais.

Abramovay (2010) considera que a análise institucional é uma possibilidade de realizar pesquisas empíricas sobre o fenômeno territorial do desenvolvimento e que a própria noção de território nos estudos de desenvolvimento abre um campo para a cooperação entre as ciências sociais e naturais por meio do conceito de sistemas socioecológico (BERKES; FOLKE, 1998; MAHON; MCCONNEY; ROY, 2008). A delimitação espacial dos sistemas socioecológico pode se sobrepor à categoria de território, que é definida a partir da construção institucional dos atores 
(CARRIÈRE; CAZELLA, 2006). O arcabouço conceitual proposto neste artigo enriquece a análise de desenvolvimento ao explicar as mudanças das "regras-em-uso" (OSTROM, 2005) de gestão dos recursos de uso comum em estreita sintonia com as operações de desenvolvimento realizadas no território.

\section{Metodologia}

O arcabouço conceitual apresentado é resultante de dados empíricos, coletados e analisados através da metodologia proposta pela teoria fundamentada na prática (HEATH; COWLEY, 2004). Inicialmente concebida por Glaser e Strauss (1967), a Teoria Fundamentada na Prática (TFP) tem o objetivo de gerar ou descobrir uma teoria. A teoria fundamentada na prática (Grounded Theory) oferece um contraponto entre as pesquisas desinformadas teoricamente e as teorias desenvolvidas com níveis conceituais altamente abstratos (GOULDING, 1998). Segundo Suddaby (2006), a teoria fundamentada na prática descreve a emergência de uma teoria com base em quão bem os dados se ajustam às categorias conceituais identificadas por um observador, quão bem essas categorias explicam ou predizem as interpretações e quão relevantes são as categorias para as questões centrais que estão sendo observadas.

O trabalho de campo que fundamenta o arcabouço conceitual foi realizado ao longo de 154 dias distribuídos em dois anos e oito meses (fevereiro de 2011 a setembro de 2013). No nível local, foram estudadas duas comunidades, uma de cada município que compõe a Baía da Ilha Grande: Tarituba (município de Paraty) e Mambucaba (município de Angra dos Reis). O marco temporal inicial foi o Decreto-lei $\mathrm{n}^{\mathrm{O}} 221$, de 1967, que dispõe sobre a proteção e estímulos à pesca. As etapas da pesquisa foram compostas pelo levantamento de dados, identificação dos vetores de mudança no nível comunitário e análise das: (1) respostas dos pescadores artesanais (inovações técnicas); (2) consequências para a pesca local; e (3) mudanças nas declarações de regras da pesca artesanal. Os procedimentos de coleta de dados foram descritos em De Freitas e Seixas (2014) e De Freitas e Seixas, Seixas (2015). De forma geral, os métodos de pesquisa incluíram a análise das fontes documentais, observação direta em arenas de tomada de decisão, observação participante em pescarias, entrevistas semiestruturadas (39) e abertas (110). 


\title{
Instituições para o estudo da mudança em territórios e recursos de uso comum
}

A análise que empreendo é baseada no trabalho de North (1990), que considera ser o papel das instituições reduzir as incertezas pelo fornecimento de uma estrutura estável para a interação humana:

\begin{abstract}
São as instituições que possibilitam a vida diária prosseguir com um mínimo de negociação repetitiva; instituições reduzem custos transacionais. Mas, é também a existência de instituições que fornecem a dimensão reguladora do contato humano diário que se torna o status quo e que por isso se torna o foco da atenção quando decisões coletivas devem ser tomadas para responder a novas escassezes, novos sabores, preferências e oportunidades (BROMLEY, 1989, p. 39, grifo e tradução minhas).
\end{abstract}

Para abordar a mudança institucional, é necessário ter uma compreensão do que são os custos transacionais e qual sua importância no debate. Os custos transacionais são "(...) o custo de medir os atributos valoráveis do que está sendo trocado e os custos de proteger os direitos, policiar e de fazer cumprir os acordos" (NORTH, 1990, p. 27, tradução minha). Na literatura sobre custos transacionais, é preciso atentar para os mecanismos de cumprimento das regras, pois sua estrutura, a frequência e severidade da imperfeição desempenham um papel crucial nos custos transacionais e nas formas que os contratos assumem. Para atingir uma fiscalização efetiva sem a presença formal e constante de uma terceira parte, é preciso de outros incentivos para chegar a acordos, como a reputação, várias formas de lealdade, crenças comuns que ligam grupos minoritários e compromissos ideológicos (NORTH, 1990). Essas instituições têm baixo custo transacional e constituem elementos presentes na ação coletiva desempenhada em diferentes casos de sistemas auto-organizados:

[...] criando um ambiente institucional que favoreça comprometimentos, permite um complexo arcabouço institucional de regras formais, restrições informais e fiscalização que, juntos, tornam possível transações de baixos custos. (NORTH, 1990 , p. 58, tradução minha).

O sentido atribuído à impessoalidade na mudança institucional está relacionado com a complexa troca impessoal necessária para haver estabilidade política e para capturar os ganhos econômicos potenciais da tecnologia moderna (NORTH, 1990). A exemplo da transferência de tecnologia, agências de desenvolvimento muitas vezes implementam pacotes institucionais trazidos 
de outras regiões por experts. Os resultados desse processo de construção de instituições, denominado por Evans (2004) de "monocultura institucional”, são sistemas com pouca variedade de regras formais em ambientes com elevados níveis de diversidade ecológica. Desenvolver melhores ferramentas para o estudo da mudança institucional é um passo importante para reduzir a ênfase da monocultura institucional dominante no pensamento das ciências sociais e agências de desenvolvimento (OSTROM; BASURTO, 2011).

No início dos anos 2000, Agrawal (2002) constatou que os estudos sobre recursos de uso comum avançaram nas análises das estruturas institucionais, porém pouco se sabia sobre sua dinâmica (BERKES, 2002) operando em diferentes escalas (CASH et al., 2006). Desse modo, as análises recentes sobre mudança institucional vêm recebendo um crescente aprimoramento teóricometodológico (NORTH, 2005; OSTROM, 2005, 2007; OSTROM; BASURTO, 2011). Segundo Jansen (2005), o estudo da mudança institucional é difícil, pois instituições são criadas, selecionadas, armazenadas, fiscalizadas, mudadas e excluídas por atores distintos em diferentes escalas (e.g., temporal, espacial e organizacional).

A mudança institucional baseia-se nas interações que ocorrem entre organizações e instituições (NORTH, 1990) ao longo do tempo. A existência e a operação das organizações dependem de um conjunto de instituições que define o que e como as organizações vão fazer, como vão se relacionar com o mundo externo e como vão remunerar seus empregados (BROMLEY, 1989). Organizações são grupos de indivíduos ligados por alguma proposta comum para atingir objetivos. As organizações alteram a estrutura institucional, que é consequência das oportunidades resultantes do conjunto de restrições existentes (NORTH, 1990). Na mudança institucional é preciso identificar quais são os incentivos para descumprir uma regra e punir os descumprimentos (AXELROD, 1986).

Ostrom (1992) considera a existência de um banco ou um "capital" de instituições que podem estimular a criatividade e servirem para iluminar a dinâmica das instituições. Segundo Ostrom (2005), é impossível conduzir uma análise completa do desempenho esperado das mudanças potenciais nas regras visando aprimorar os resultados a serem atingidos. Isso porque a elevada quantidade de combinações possíveis entre as regras conduziria o analista institucional a uma complexidade inviável de lidar em termos práticos. A construção de regras pelos usuários lida com as incertezas do mercado e as características das interações entre os recursos e o meio biofísico. Representantes do poder público e os usuários do recurso podem tentar mudar o desempenho de sistemas de recursos de uso comum pela mudança em uma ou mais regras em um 
processo adaptativo. Os agentes usam de sua persuasão e razão para criar regras melhores, porém as regras que serão usadas sempre envolverão experimentação (OSTROM, 2005).

Os resultados da mudança institucional dependem do conhecimento e das habilidades de comunicação e aprendizado das organizações como jogadores (NORTH, 1990). A decisão de participar dos processos de mudança institucional é tomada por atores cujo comportamento é guiado por ideias, ideologias, mitos, dogmas, preconceitos (NORTH, 1993) e percepções dos custos e benefício envolvidos (NORTH, 1990). Se a soma dos custos esperados pelos atores exceder o incentivo para mudança, nenhum ator vai investir tempo e recursos necessários para criar instituições (OSTROM, 1990). Isso significa que haverá um esforço para mudar o status quo se os benefícios percebidos pelas organizações em alterar as regras superar os custos percebidos (BROMLEY, 1989; NORTH, 1990; OSTROM, 1990).

Os mecanismos de mudança institucional podem ser amplamente classificados em autoconscientes (baseados na habilidade humana para aprender) e inconscientes (OSTROM; BASURTO, 2011). Segundo Ostrom e Basurto (2011), no estudo da mudança institucional é preciso estar atento às condições que propiciam o aumento no aprendizado e produzem a evolução dos sistemas de regras, entendidas como mecanismos de informação-transformação (OSTROM, 2005).

A relevância que teóricos da trajetória de desenvolvimento e da mudança institucional conferem à categoria da aprendizagem indica que há perspectivas de integração entre essas escolas. $\mathrm{Na}$ mudança institucional, a aprendizagem ocorre por tentativa e erro, processo no qual as estratégias mais efetivas são retidas e, por observação, aqueles que apresentam desempenho inferior imitam as estratégias dos que estão se saindo melhor (AXELROD, 1986). O estudo de Sabourin (2001) sobre a trajetória de desenvolvimento do Agreste paraibano ilustra essa compreensão da mudança institucional:

De fato, para as práticas agropecuárias, entre outras, existe uma norma técnica de base, às vezes minimalista e comum ao grupo, que evolui lentamente, até por conta da pressão social dentro do grupo. As mudanças provêm de fatores externos ou, internamente, de práticas consideradas como "desvios" a essa norma. Eventualmente, depois de certo tempo de "observação", tal prática poderá substituir a norma anterior (SABOURIN, 2001, p. 7, tradução minha). 
A seguir, serão apresentadas as variáveis que compõem o arcabouço conceitual e discutido como elas se articulam na análise da trajetória de desenvolvimento de territórios através da mudança institucional.

\section{Proposta de um arcabouço conceitual para a análise da trajetória de desenvolvimento}

Os processos envolvidos no estudo da trajetória de desenvolvimento de territórios foram sintetizados em uma proposta de arcabouço conceitual (Figura 01) focado na mudança institucional. O modelo é centrado na mudança institucional, sendo que as variáveis circuladas (vetores de mudança, inovação, consequência, regras formais e informais) constituem o foco da análise.

As instituições condicionam a organização social requerida para as inovações técnicas, que por sua vez estão diretamente associadas com a disponibilidade dos recursos naturais. As variáveis circuladas do arcabouço conceitual (vetores de mudança, inovação, consequências, regras formais e informais) constituem o foco do estudo da trajetória de desenvolvimento de territórios. Os vetores de mudança constituem um conjunto de variáveis independentes, que são considerados em contraste com o tipo específico de mudança ambiental (YOUNG, 2002). Os vetores de mudança podem ser a construção de uma rodovia ou de uma indústria, a modificação do curso de um rio e a introdução de espécies exóticas ou de novos materiais que modificam a forma de uso e apropriação aos recursos naturais de uso comum e geram mudança institucional.

Sabourin (2001) considerou como produtores e vetores de inovação o papel dos agricultores, de suas organizações e das instituições técnicas. No contexto de recursos naturais de uso comum, considero que as inovações técnicas representam as respostas adaptativas dos usuários aos vetores de mudança dos sistemas socioecológicos. As consequências estão relacionadas ao feedback gerado por um vetor de mudança para a base de recursos naturais em questão. 


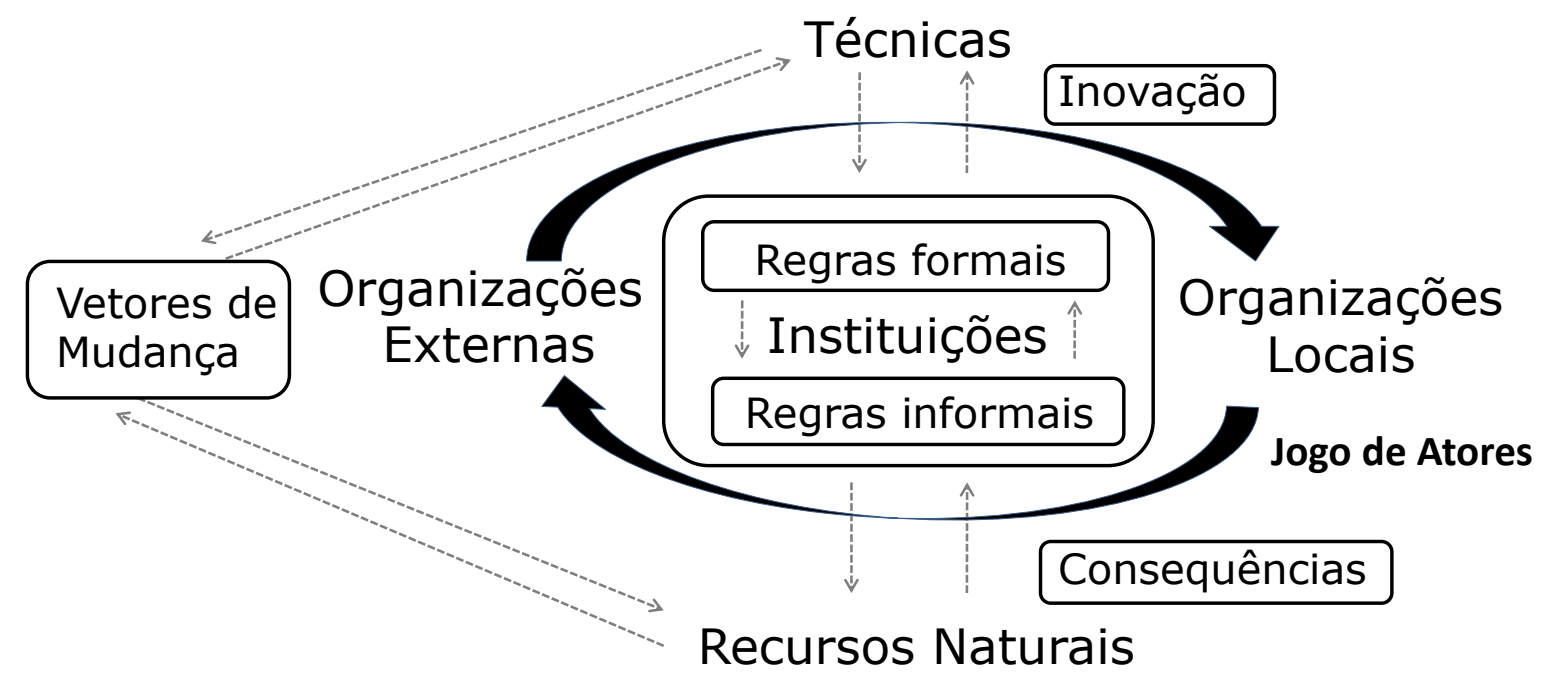

FIGURA 01 - ARCABOUÇO CONCEITUAL PARA ANÁLISE DA TRAJETÓRIA DE DESENVOLVIMENTO DE TERRITÓRIOS.

FONTE: De Freitas (2014).

Consideremos o caso da pesca na Baía da Ilha Grande (DE FREITAS, 2014). Quando os barcos a motor foram introduzidos, foram possíveis inovações nas técnicas de pesca, como o arrasto de portas motorizado. A introdução do náilon multifilamento no lugar no náilon seda também constituiu um vetor de mudança que gerou importantes inovações técnicas, como a popularização de diferentes modelos de redes de emalhe. O aumento na quantidade de pescadores utilizando técnicas de captura mais eficientes (rede de emalhe e arrasto de portas) e com maior captura incidental contribuiu para a escassez de recursos pesqueiros (consequência) evidenciada durante a década de 1980. O aumento do turismo trouxe inovações para as comunidades, como a construção de quiosques na orla, a chegada de novos moradores e a construção de bares nas ilhas. Como consequência, as embarcações de pesca começaram a ter dupla atividade e os pescadores passam a atuar no transporte de turistas para as ilhas.

As regras ou instituições formais e informais constituem o foco da análise institucional. Instituições formais e informais estão intimamente interconectadas e sua interdependência afeta quando e como as mudanças irão ocorrer em ambos os lados (CARRUTHERS, 2012). Regras formais são restrições regulamentadas pelo Estado na forma de diplomas legais, como leis e constituições, enquanto as regras informais são restrições associadas a normas de comportamento, convenções e códigos de conduta autoimpostos (NORTH, 1990). Para North (1990), as regras 
formais incluem regras políticas (definem a hierarquia e a estrutura básica do processo decisório), as regras econômicas (definem direitos de apropriação) e os contratos (definem restrições e direitos individuais gerais e específicos). Cabe destacar que, de acordo com as regras políticas, as regras formais estão envolvidas em níveis imbricados, com níveis mais elevados, fornecendo o contexto para os níveis abaixo (OSTROM, 1990).

Em sistemas de manejo comunitário, a regulação do uso e o controle do acesso aos recursos naturais são fundamentalmente baseados em regras informais (ver RUTTAN, 1998). Por meio de extensa documentação de estudos de caso e em experimentos realizados em laboratório (OSTROM, 1990, 2010; OSTROM; BASURTO, 2011; POTEETE; JANSSEN; OSTROM, 2010), foi mostrado que os usuários de recursos que possuem relativa autonomia para desenvolver suas próprias regras sobre como governar e gerir os recursos de uso comum frequentemente atingem resultados econômicos melhores e mais equitativos. No Saco de Tarituba, por exemplo, os pescadores reivindicaram a formalização da proibição do arrasto de portas junto a Prefeitura de Paraty. A Prefeitura atendeu ao pedido dos pescadores e esta é uma regra que conta com o apoio local para operacionalizar seu cumprimento (DE FREITAS, 2014).

Regras informais estão associadas ao sentido atribuído às normas por Axelrod (1986), ou seja, como sendo vinculadas aos comportamentos coordenados para regulação de conflitos sem a existência de uma autoridade central. Dessa forma, regras se distinguem de normas por terem uma sanção expressa em detrimento do seu não cumprimento (CRAWFORD; OSTROM, 1995). As mudanças nas instituições informais são difíceis de serem detectadas e sua transmissão ocorre através da experiência pessoal ou de um informante (CARRUTHERS, 2012). Essa diferença (formal e informal) opera mais como fins de vários contínuos do que como uma simples dicotomia (CARRUTHERS, 2012, p. 7, tradução minha):

[...] regras formais são explícitas, enquanto informais são implícitas; regras informais são mais flexíveis do que as formais; regras formais são precisas, enquanto que as informais são vagas; regras formais são escritas, enquanto as informais não são.

Interessa para a análise institucional descrever as regras em uso, uma vez que algumas regras escritas podem não ser seguidas, enquanto outras regras não escritas podem ser extremamente efetivas (OSTROM, 1990, 2005). Essa perspectiva sobre a análise institucional, 
conhecida nas ciências sociais como neoinstitucionalismo ${ }^{2}$, contrasta com os estudos restritos às regras formais, amplamente realizados no passado (YOUNG, 2002). Outro pressuposto desta análise se refere ao peso das identidades dos atores nos processos de decisão política, em detrimento do papel conferido aos interesses pessoais no modelo do ator racional (FREY, 2000).

Como a análise da criação e das mudanças nas regras recai sobre as interações entre organizações, essa perspectiva foi incorporada ao arcabouço conceitual. A interação entre as organizações (locais e externas) para alterar as regras é analisada através do jogo de atores. Esta análise busca explicar a gênese dos conflitos, representações sociais e cooperações entre os atores para promover mudanças no status quo ajustadas ao desenvolvimento territorial sustentável (VIEIRA; CAZELLA, 2006). As variáveis do jogo de atores podem ser organizadas através do arcabouço Institutional Analysis and Development - IAD (OSTROM, 2005), em que merece destaque o conceito de situação de ação.

Atores em situação de ação representam um sistema de relações para influenciar e dirigir a formulação e implementação de políticas públicas (OSTROM, 1990). O IAD procura classificar as regras de acordo com o seu impacto direto sobre as partes de uma situação de ação (OSTROM, 2005). A situação de ação pode ser entendida como um jogo formal que é composto por partes internas (OSTROM, 2010). Segundo Ostrom (2005, p. 17): “As regras podem ser pensadas como um conjunto de instruções para criação de uma situação de ação em um ambiente particular”. Nesse sentido, o jogo de atores analisa as interações por meio da evolução das dinâmicas conflitivas e cooperativas (coalizões) entre os atores sociais que participam direta ou indiretamente das situações de ação. O conflito pode desencadear processos de mudança institucional, sendo analisado tanto como uma categoria explicativa quanto como propulsor de mudanças sociais (FERREIRA, 2005). Dada a heterogeneidade e as assimetrias de poder existentes na sociedade, o conflito não é um problema com uma possibilidade definitiva de resolução (FERREIRA, 2004).

A análise institucional proposta pelo IAD reconhece regras de posição e de escolha coletiva que indicam a hierarquia entre os atores no processo decisório. No entanto, as explicações derivadas desse modelo estão normalmente associadas com a compreensão de fatores relacionados com a obediência das regras (e.g., POLLNAC et al., 2010; POTEETE, JANSSEN; OSTROM, 2010).

\footnotetext{
${ }^{2}$ Consultar Frey (2000) e Souza (2006) para uma discussão sobre as diferenças do neoinstitucionalismo para outros modelos de formulação e análise de políticas públicas.
} 
Nesse sentido, a análise do jogo de atores complementa aspectos de ordem explicativa do arcabouço $\mathrm{IAD}$ relativa às relações de poder e aos mecanismos de dominação existentes nas arenas.

\section{Conclusão}

Apresentei um arcabouço conceitual para o estudo da trajetória de desenvolvimento de territórios baseado nas interações entre instituições e organizações. O foco sobre as atividades produtivas, presente na perspectiva da trajetória de desenvolvimento de territórios, foi complementado pela abordagem das mudanças nas regras em uso, trazido pela mudança institucional. $\mathrm{O}$ arcabouço conceitual contempla a retroalimentações entre vetores de mudança, inovações técnicas, consequências para os recursos naturais na passagem das regras informais para as regras formais. Na manipulação do arcabouço, é recomendado que a análise das interações específicas existentes entre as organizações internas e externas, expressas pelo jogo de atores, utilize as variáveis presentes em outros modelos, como o Institutional Analysis and Development (OSTROM, 2005).

A mudança institucional pode representar uma resposta à crítica de Abramovay (2010) sobre os estudos de desenvolvimento territorial que se atém à presença ou ausência de elementos favoráveis à dinâmica de desenvolvimento de certas regiões. A abordagem tradicional da trajetória de desenvolvimento focaliza nas atividades produtivas de uma região, diferindo da mudança institucional que aborda as mudanças nas regras em uso em uma situação de ação. No entanto, enquanto a análise institucional volta seu foco para as alterações específicas nos tipos de regras, ela somente tangencia o efeito dessas mudanças para os demais atores sociais que compartilham o território. A abordagem da trajetória de desenvolvimento pode indicar que atividades competem e/ou cooperam sob condições específicas. Assim, uma análise integrada da trajetória de desenvolvimento e da mudança institucional permite delinear políticas públicas que contemplem a especificidade da situação da ação analisada na sua interação com as demais atividades praticadas pelos atores sociais que compartilham o território.

A partir de um diálogo entre as categorias de sistema socioecológico e território, o arcabouço conceitual busca inserir a gestão de recursos de uso comum em um cenário de desenvolvimento territorial sustentável. Para além da curiosidade científica acerca da mudança institucional, é necessário ressaltar a importância que a temática tem para o processo de formulação 
de políticas públicas em tempos de crise socioecológica. Assim como Abramovay (2010) e Carrière e Cazella (2006), consideramos que a mobilização de estudos de caso utilizando a análise institucional pode contribuir para os avanços nas pesquisas sobre o fenômeno territorial do desenvolvimento.

\section{Agradecimentos}

Sou grato ao Programa CAPES - Ciências do Mar (Projeto Gestão Integrada e Compartilhada de Territórios Marinho-Costeiros: implicações para a pesca artesanal e conservação da biodiversidade) pelo financiamento da pesquisa de campo e concessão da bolsa de estudos. Agradeço também ao International Development Resources Center (IDRC - Canadá) pelo apoio na participação em eventos durante o doutorado. Estendo meus agradecimentos às contribuições dos membros do Grupo de Pesquisa em Conservação e Gestão de Recursos de Uso Comum (CGCommons) do Núcleo de Estudos e Pesquisas Ambientais (Nepam) da Universidade Estadual de Campinas (Unicamp), especialmente à Luciana Araújo, que revisou uma versão anterior deste artigo, à minha orientadora (Cristiana S. Seixas) e co-orientadora, Sônia R. da Cal Seixas.

\section{Referências}

ABRAMOVAY, R. Por uma teoria dos estudos territoriais. In: Vieira, P. F.; CAZELLA, A.; CERDAN, C.; CARRIÉRE, J-P. (Eds.). Desenvolvimento territorial sustentável no Brasil: subsídios para uma política de fomento. Florianópolis: Aped, 2010.

AGRAWAL, A. Common resources and institutional sustainability. In: OSTROM, E.; DIETZ, T.; DOLSAK, N.; STERN, P.; STONICH, S.; WEBER, E. U. (Eds.). The drama of the Commons. Washington: Natl. Acad. Press, 2002.

ANDION, C. Atuação das ONGs nas dinâmicas de desenvolvimento territorial sustentável no meio rural de Santa Catarina: os casos da Apaco, do Centro Vianei de Educação Popular e da Agreco. Florianópolis: Tese de Doutorado. Programa Interdisciplinar em Ciências Humanas, UFSC, 2007. 
AXELrOD, R. An evolutionary approach to norms. The American Political Science Review. Denton, v. 80, n. 4, p. 1095-1111, 1986.

BERDEGUÉ, J.; BEBBINGTON, A.; ESCOBAL, J.; FAVARETO, A.; FERNÁNDEZ, I.; OSPINA, P.; RAVNBORG, H. MUNK; AGUIRRE, F.; CHIRIBOGA, M.; GÓMEZ, I.; GÓMEZ, L.; MODREGO, F.; PAULSON, S.; RAMÍREZ, E.; SCHEJTMAN, A.; TRIVELLI, C. Territorios en movimiento. Dinámicas territoriales rurales en América Latina. Santiago: Rimisp, 2012. (Programa Dinámicas territoriales rurales, Documento de trabajo, n. 110).

BERKES, F. Cross-scale institutional linkages: perspectives from the bottom up. In: OSTROM, E.; DIETZ, T.; DOLSAK, N.; STERN, P.; STONICH, S.; WEBER, E. U.(Eds.). The drama of the Commons. Washington: Natl. Acad. Press, 2002.

BERKES, F.; FOLKE, C. Linking social and ecological systems for resilience and sustainability. In: BERKES, F.; FOLKE, C. (Orgs.). Linking social and ecological systems. Cambridge: Cambridge University Press, 1998.

BROMLEY, D. W. Economic interests and institutions: the conceptual foundations of public policy. New York: Basil Blackwell, 1989.

CARRIÈRE, J.-P.; CAZELLA, A. A. Abordagem introdutória ao conceito de desenvolvimento territorial. Eisforia, Florianópolis, v. 4, n. especial, 2006.

CARRUTHERS, B. G. Institutional dynamics: when is change "real change"? Comparative Historical Social Science (CHSS). Working Paper Series, n. 12, 2012.

CERDAN, C.; VIEIRA, P. F.; POLICARPO, M.; VIVACQUA, M.; CAPELLESSO, A.; RODRIGUES, H. C.; MARTINEL, B.; CORDEIRO, E.; LESAGE, A.; MEYNARD, F.; PEDROSA, A.; ADRIANO, J.; LEONEL, M.; FERREIRA, M. Desenvolvimento territorial sustentável na zona costeira do Estado de Santa Catarina, Brasil. Santiago: Rimisp, 2011. (Programa Dinámicas Territoriales Rurales, Documento de Trabajo, n. 87).

CRAWFORD, E. S.; OSTROM, E. A grammar of institutions. The American Political Science Review, Denton, v. 89, n. 3, p. 582-600, 1995.

DE FREITAS, R. R. Implicações de políticas de conservação e desenvolvimento na pesca artesanal costeira em uma Área Marinha Protegida da Baía da Ilha Grande. Tese de Doutorado. Unicamp, Nepam, Campinas, 2014.

DE FREITAS, R. R.; SEIXAS, C. S. Socio-ecological changes in artisanal fishery of two communities adjacent to a Brazilian marine reserve. In: 2nd World Small-Scale Fisheries Congress. Mérida, 2 nd World Small-Scale Fisheries Congress Proceedings, 2014.

DE FREITAS, R. R.; SEIXAS, C. S. ; SEIXAS, S. R. C. Implicações de políticas para a pesca artesanal costeira na trajetória de desenvolvimento da Baía da Ilha Grande (Rio de Janeiro). In: QUADROS, J; SANTOS, P. A. (Orgs.). Diálogos interdisciplinares em desenvolvimento 
territorial sustentável: políticas, ecologias e saberes. Matinhos: Universidade Federal do Paraná, 2015. (v. 1)

EVANS, P. Development as institutional change: the pitfalls of monocropping and the potentials of deliberation. Studies in Comparative International Development, Providence, v. 39, n. 4, p. 30$52,2004$.

FERREIRA, L. C. Dimensões humanas da biodiversidade: mudanças sociais e conflitos em torno de área protegidas no Vale do Ribeira, SP. Ambiente e Sociedade, São Paulo. v. 7, n. 1, p. 47-66, 2004.

FERREIRA, L. C. Conflitos sociais e uso de recursos naturais: breves comentários sobre modelos teóricos e linhas de pesquisa. Política e Sociedade, Florianópolis, v. 7, p. 105-118, 2005.

FLORES, M. X. Da solidariedade social ao individualismo: um estudo sobre o desenvolvimento do Vale dos Vinhedos na Serra Gaúcha. Tese de Doutorado. Programa de Pós-Graduação em Sociologia Política, UFSC, Florianópolis, 2007.

FREY, K. Políticas públicas: um debate conceitual e reflexões referentes à prática da análise de políticas públicas no Brasil. Planej. e Políticas Públicas, Brasília, v. 21, p. 211-259, 2000.

GOULDING, C. Grounded theory: the missing methodology on the interpretivist agenda. Qualitative market research: an international journal, Buckinghamshire, v. 1, n. 1, p. 50-57, 1998.

SOUZA, C. Políticas públicas: uma revisão da literatura. Sociologias, Porto Alegre. v. 16, p. 20-45, 2006.

SUDDABY, R. From the editors: what grounded theory is not. Academy of Management Journal, London, v. 49, n. 4, p. 633-642, 2006.

HEATH, H.; COWLEY, S. Developing a grounded theory approach: a comparison of Glaser and Strauss. International Journal of Nursing Studies, v. 41, p. 141-150, 2004.

MAHON, R.; MCCONNEY, P.; ROY, R. N. Governing fisheries as complex adaptive systems. Marine Policy, Cardiff, v. 32, p. 104-112, 2008.

NORTH, D. C. Institutions, institutional change and economic performance. Cambridge: Cambridge University Press, 1990.

NORTH, D. C. What do we mean by rationality? Public Choice, Logan, v. 77, n. 1, p. 159-162, 1993.

NORTH, D. C. Understanding the process of economic change. Princeton: Princeton University Press, 2005.

OSTROM, E. Governing the Commons. Cambridge: Cambridge University Press, 1990. 
OSTROM, E. Crafting institutions for self-governing irrigation systems. San Francisco: ICS Press, 1992.

OSTROM, E. Understanding institutional diversity. Princeton: Princeton University Press, 2005.

OSTROM, E. A diagnostic approach for going beyond panacea. PNAS, Washington, v. 104, n. 39, p. 15181-15187, 2007.

OSTROM, E.; BASURTO, X. Crafting analytical tools to study institutional change. Journal of Institutional Economics, Hertfordshire, v. 7, n. 3, p. 317-343, 2011.

POLLNAC, R.; CHRISTIE, P.; CINNER, J. E.; DALTON, T.; DAW, T. M.; FORRESTER, G. E.; GRAHAM, N. A. J.; McCLANAHAN, T. R. Marine reserves as linked socialecological systems. PNAS, Washington, v. 107, n. 43, p. 18262-18265, 2010.

POTEETE, A. R.; JANSSEN, M.; OSTROM, E. Working together: collective action, the Commons, and multiple methods in practice. Princeton: Princeton University Press, 2010.

RUTTAN, L. M. Closing the Commons: cooperation for gain or restraint? Human Ecology, New York, v. 26, n. 1, p. 43-66, 1998.

SABOURIN, E.; CARON, P.; SILVA, C. G. da. Estudo das trajetórias de desenvolvimento: contribuição metodológica para a análise das dinâmicas agrárias. Ateliers de Caravelle, Toulouse, v. 7, p. 53-72, 1996.

SABOURIN, E. Aprendizagem coletiva e construção social do saber local: o caso da inovação na agricultura familiar da Paraíba. Estudos sociedade e agricultura, Rio de Janeiro, v. 16, p. 37-61, 2001.

SILVA, P. C. G.; SABOURIN, E.; CARON, P.; HUBERT, B. Estudo de trajetórias de desenvolvimento local e construção do espaço rural no nordeste semi-árido. Agricultura Familiar, Pesquisa, Formação e Desenvolvimento, Belém, v. 1, n. 2, p. 5-27, 2000.

VIEIRA, P. H. F. Rumo ao desenvolvimento territorial sustentável: esboço de roteiro metodológico participativo. Eisforia, Florianópolis, v. 4, n. especial, p. 249-309, 2006.

VIEIRA, P. H. F.; CAZELLA, A. A. Desenvolvimento territorial sustentável em zonas rurais: subsídios para a elaboração de um modelo de análise. In: Territórios rurales en movimiento: movimientos sociales, actores y instituciones del desarrollo territorial rural. Santiago do Chile: Rimisp, 2006. Seminário Internacional.

YOUNG, O. R. The institutional dimensions of environmental change: fit, interplay, and scale. Cambridge: MIT Press, 2002.

Artigo recebido em 15/11/2016. Aceito para publicação em 14/12/2016. 